SCIREA Journal of Materials

http://www.scirea.org/journal/Materials

May 17, 2021

Volume 6, Issue 2, April 2021

SCIREA

\title{
Controlled Drug Release from Sodium Alginate Film
}

\section{Dosage Forms}

\author{
Yoshifumi Murata*, Sayaka Kimura, Kyoko Kofuji, Chieko Maida \\ Faculty of Pharmaceutical Science, Hokuriku University, Ho-3, Kanagawa-machi, Kanazawa \\ 920-1181, Japan \\ Email address: y-murata@hokuriku-u.ac.jp (Yoshifumi Murata) \\ *Corresponding author
}

\begin{abstract}
Film dosage forms (FDs) prepared using water-soluble polymers can efficiently deliver drugs to local disease sites. In the present study, we prepared FDs by incorporating a model drug rebamipide into a film made of sodium alginate (Alg-Na), comprising an alginic acid hydrolysate and $\alpha$-L-guluronic acid (G) block (G-block), as the film base. The drug immediately dissolved from the FDs prepared with 3\%-5\% Alg-Na, and the amount Alg-Na in the formulation scarcely affected the drug dissolution profile. The drug dissolution rate decreased, according to the increment in the amount of G-block incorporated into the FD, as the aqueous solubility of the drug decreased after G-block incorporation. The rate did not change after the sodium salt of the G-block was added to the film base. Rebamipide FDs
\end{abstract}


prepared with Alg-Na may be effective for treating oral mucositis, as the drug release rate from FDs can be controlled by modifying the composition of the film base.

Keywords: Film Dosage Form, Alginic Acid Hydrolysate, Rebamipide, Controlled Drug Release, Sodium Alginate

\section{Introduction}

Alginic acid is an algal polysaccharide that consists of $\alpha$-L-guluronic acid (G) and $\beta$-D-mannuronic acid (M). The sequence of three block structures resulting from the polymerization of $\mathrm{G}$ and $\mathrm{M}$ residues, G-, M-, and MG-block, is a dominant factor in the characteristics of the polymer $[1,2]$. The synthesis of each block is achieved via hydrolysis with a dilute $\mathrm{HCl}$ solution, and the separation of each block type in the reaction solution is achieved by controlling the $\mathrm{pH}$ of the solution. The utilization of G-block to control the drug dissolution rate from calcium-induced alginate gel beads has been investigated [3]. The sodium salt of alginic acid Alg-Na is a water-soluble polysaccharide widely utilized as a food ingredient and/or as an excipient in drug products, owing to its polymeric properties and safety on oral intake [4, 5]. In addition, Alg-Na has gastroprotective action [6-8].

Rebamipide (RM) has been utilized as a gastroprotective drug against gastric ulcers $[9,10]$, and it is locally administered for oral mucositis or ulcers, such as mouthwash $[11,12]$. Although the mode of administration for alleviating these complications is simple, it is difficult to control the dose of RM during mouth washing. As film dosage forms (FDs) are prepared with water-soluble polymers, they swell and disintegrate in a small amount of body fluid, such as saliva; therefore, FDs can be an efficient tool for delivering drugs to oral disease sites [13-15]. We have studied FDs prepared with natural polysaccharides, such as chondroitin 
sulfate or pectin, as a film base by simple methods that do not require dissolution in organic solvents $[16,17]$. In the case of FDs prepared with Alg-Na incorporating RM, the disintegration profile of the FD differed according to the molecular-weight of Alg-Na [18]. However, the disintegration of the FD film matrix did not affect the drug dissolution rate.

Controlling the drug dissolution rate from such formulations, including the oral disintegration films, is key in achieving efficient drug delivery to the disease site [19]. In this study, RM-loaded FDs were prepared via the casting method using an Alg-Na-containing G-block as the film base from which the dissolution rate of the drug in limited medium could be controlled.

\section{Materials and Methods}

Material. Three species of low-molecular-weight Alg-Na, Alg-A (IL-1, Kimica Co., Tokyo, Japan), Alg-B (IL-1G, Kimica Co.), and Alg-C (I-1G, Kimica Co.) were used as base films. A high-molecular-weight Alg-Na (300 cps, Nacali Tesque Inc., Kyoto, Japan) was used to prepare the G-block. RM was purchased from Tokyo Chemical Industry Co., Ltd. (Tokyo, Japan). Hydroxylamine was purchased from Wako Pure Chemical Industries, Ltd. (Osaka, Japan). Water-soluble carbodiimide and 1-cyclohexyl-3-(2-morpholinoethyl)carbodiimide metho-p-toluenesulfonate (CMEC) were purchased from Aldrich Chemical Co. (Milwaukee, WI, USA). All other chemicals were of reagent grade and were obtained from commercial sources.

Preparation of the G-block and its Sodium Salt. High-molecular-weight Alg-Na was partially hydrolyzed $\left(0.2 \mathrm{M} \mathrm{HCl}, 2 \mathrm{~h}, 100{ }^{\circ} \mathrm{C}\right)$, and the G-block was separated using methods described in Haug et al. [20]. The sodium salt of the G-block was obtained as follows: the G-block was suspended in ion-exchanged water to which $\mathrm{NaOH} 0.2 \mathrm{M}$ was added to the suspension with stirring. The $\mathrm{pH}$ of the solution was then adjusted to approximately 7 to produce the sodium 
salt. Ethanol was added to provoke the formation of a white precipitate in the solution that was collected after centrifugation at $1,900 \times g(2100$ model; Kubota Co., Tokyo, Japan $)$ for 5 min; this was then washed with ethanol three times and dried. The white block obtained was ground with a pestle and sieved (mesh size, $75 \mu \mathrm{m}$ ) before being used in subsequent experiments.

FD Preparation. The FD was prepared as follows: $10 \mathrm{~g}$ of the base solution containing RM was dispersed in deionized water to prepare the film base. The mixture by sonicated and poured (3 g each) into individual plastic Petri dishes (diameter, $54 \mathrm{~mm}$ ). The dishes were kept at $40{ }^{\circ} \mathrm{C}$ for $24 \mathrm{~h}$. The circular films formed were transferred into a desiccator. The thickness of each film was measured at 10 points using a micrometer (CLM1-15QM; Mitutoyo, Kawasaki, Japan), with a set pressure of $0.5 \mathrm{~N}$. Three films were measured, and the mean thickness was calculated.

Drug Dissolution Test. One of the films was placed in a plastic dish, and $10 \mathrm{~mL}$ of physiological saline, preheated to $37^{\circ} \mathrm{C}$, was added. The dish was then shaken at $300 \mathrm{rpm}$ in an incubator (SI-300; As One Co., Osaka, Japan) at $37^{\circ} \mathrm{C}$. Aliquots were withdrawn $(0.3 \mathrm{~mL})$ at regular intervals using a plastic syringe and filtered through a syringe-driven filter unit (pore size: $0.45 \mu \mathrm{m})$. An equal volume $(0.3 \mathrm{~mL})$ of physiological saline at $37^{\circ} \mathrm{C}$ was added to the dish in the incubator to maintain a constant volume. Next, $80-\mu \mathrm{L}$ aliquots of the filtered sample solution were placed in micro test tubes $(1.5 \mathrm{~mL}) ; 720 \mu \mathrm{L}$ of methanol was added to precipitate the polysaccharide dissolved from the dosage form. The samples were mixed and centrifuged $(7,700 \times g, 5$ min; H-1300; Kokusan Co., Saitama, Japan). The supernatant was then injected into the high-performance liquid chromatography (HPLC) stationary column. Each test was performed in triplicate.

RM Assay. The HPLC system used (Hitachi Co., Tokyo, Japan) consisted of a pump (L-2130), UV detector (L-2400), autosampler (L-2200), and chromate-integrator (D-2500) connected to 
a packed column $(150 \mathrm{~mm} \times 4.6 \mathrm{~mm}$, Cosmosil 5C18-MS-II, Nacalai Tesque, Inc.). To determine the RM concentration, the assay was performed at ambient temperature using a mobile phase consisting of $20 \mathrm{mM}$ potassium phosphate buffer (pH 6.8) and methanol (12:13) at a flow rate of $1.0 \mathrm{~mL} / \mathrm{min}$ [21]. The detector wavelength was set to $230 \mathrm{~nm}$.

Film Disintegration Test. The sample solution was isolated using the method described section 2.4. Aliquots $(0.1 \mathrm{~mL})$ of the filtered solution were combined with $0.9 \mathrm{~mL}$ of ion-exchanged water in test tubes and mixed in a vortex mixer. The amount of Alg-Na in each sample solution $(1 \mathrm{~mL})$ was measured using the method described below in section 2.7. Each test was performed in triplicate.

Alg-Na Assay in a Colorimeter. The reagent solutions used were $20 \mathrm{mM}$ hydroxylamine in ion-exchanged water and $0.1 \mathrm{M} \mathrm{CMEC} \mathrm{in} 2 \%$ pyridine- $\mathrm{HCl}$ buffer $(\mathrm{pH} 5.0)$. Aliquots $(1 \mathrm{~mL})$ of hydroxylamine and CMEC were added to $1 \mathrm{~mL}$ of the sample solution, and each mixture underwent mixing in a vortex mixer. Each mixture was incubated at $40{ }^{\circ} \mathrm{C}$ for $20 \mathrm{~min} ; 20 \mathrm{mM}$ $\mathrm{FeCl}_{3}$ in $0.1 \mathrm{M} \mathrm{HCl}(3 \mathrm{~mL})$ was added to the mixture. The absorbance of the solution in a quartz cell (1-cm light path) was measured at $480 \mathrm{~nm}$ using a spectrophotometer (UV-1200; Shimadzu, Kyoto, Japan). The absorbance was normalized to that of the reagent blank. For each test, a calibration curve was constructed using a fresh set of Alg-Na standards.

Solubility of RM. The solubility of RM was measured in water-containing additives. RM was added to the test solution, and the mixture was shaken at $37^{\circ} \mathrm{C}$ for $24 \mathrm{~h}$. The suspension was removed using a plastic syringe (Terumo Co., Tokyo, Japan), preheated to $37^{\circ} \mathrm{C}$, and filtered using a syringe-driven filter unit (Millex-HV, pore size: $0.45 \mathrm{~mm}$, Millipore Co., Danvers, MA, USA). The solution $(0.1 \mathrm{~mL})$ was diluted with methanol, mixed, and centrifuged $(7,700$ $\times g$ ) for $5 \mathrm{~min}$. The supernatant was then injected into the HPLC column.

\section{Results and Discussion}

When $2-5 \%$ low-molecular-weight Alg-Na containing RM was cast, a thin circular film was 
obtained after the solvent evaporated from the base solution. For example, an approximately $40-\mu \mathrm{m}$-thick film was formed by $4 \%$ Alg-B. The addition of the G-block to the base solution did not affect the film formation.
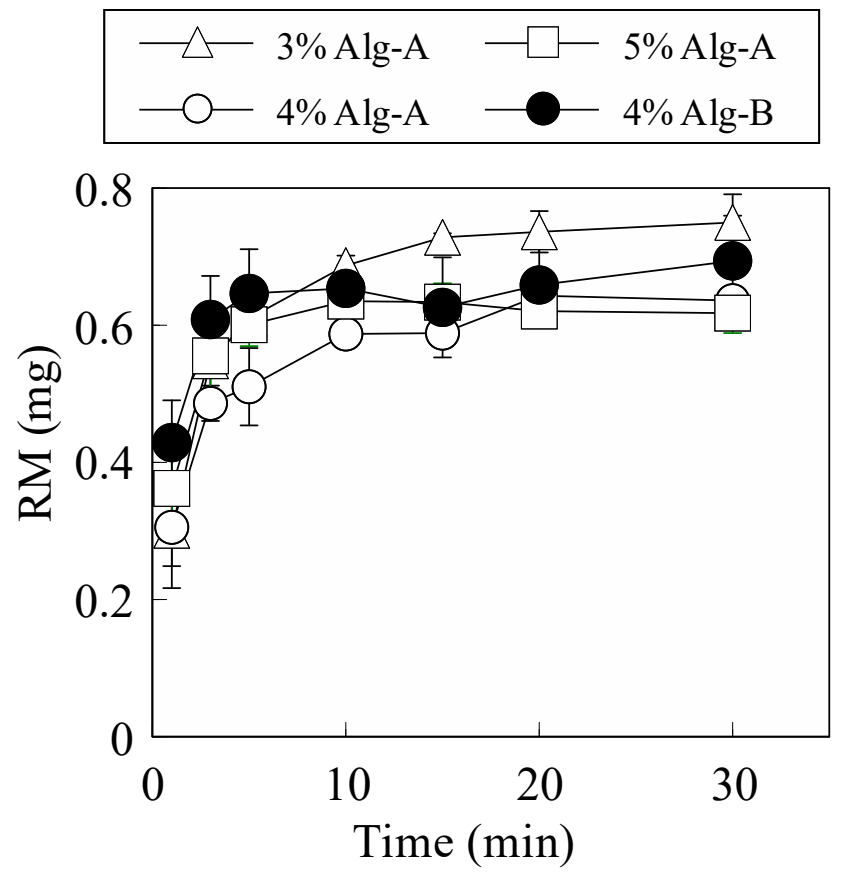

FIGURE 1: Drug release profiles of RM from FDs prepared with different concentrations of Alg-Na.

FDs prepared with Alg-Na and soaked in physiological saline at $37^{\circ} \mathrm{C}$, quickly swelled in the solution, leading to disintegration. The RM incorporated into the FD was thus released from the formulation. Figure 1 shows the drug dissolution profiles of FDs prepared with 3-5\% Alg-Na. RM dissolved quickly from the FD. For example, the amount of RM that dissolved from the FD prepared with $3 \% \mathrm{Alg}-\mathrm{A}$ at $3 \mathrm{~min}$ was $0.55 \pm 0.05 \mathrm{mg}$. Similar RM dissolution profiles were obtained from FDs prepared with 4\% and 5\% Alg-A. RM also dissolved immediately from the FD prepared with $4 \% \mathrm{Alg}-\mathrm{B}$, in which $0.61 \pm 0.06 \mathrm{mg}$ of the drug dissolved within $5 \mathrm{~min}$. These results show that the amount of drug and Alg-Na in the formulation scarcely affects the drug dissolution rate from the dosage form. 


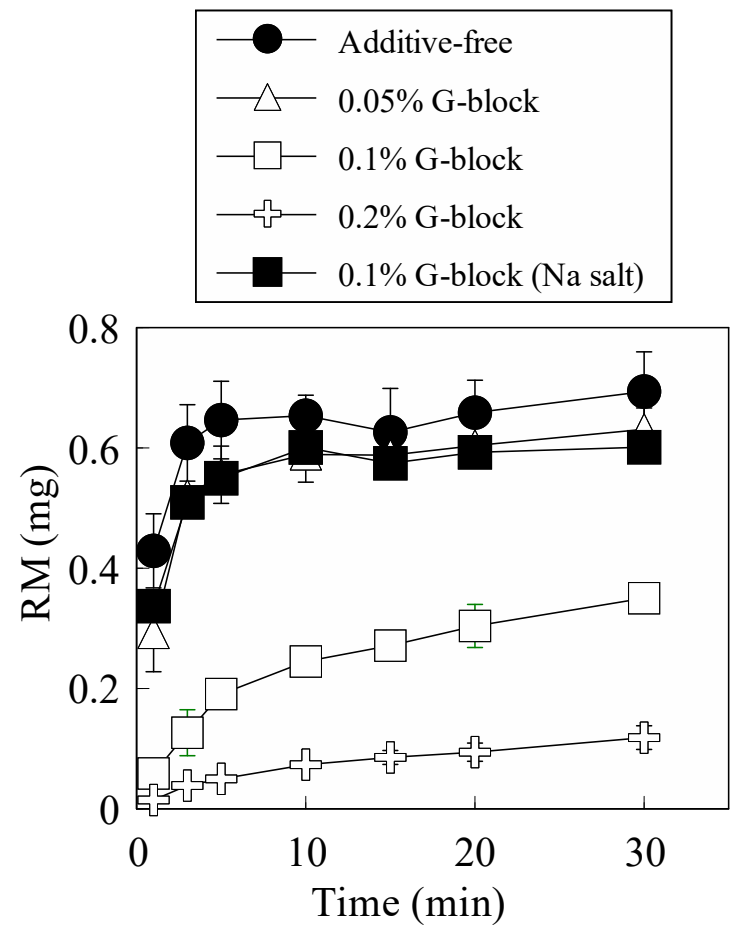

FIGURE 2: Drug release profiles of RM from FDs prepared with 4\% Alg-B containing different concentrations and types of G-block.

$$
\begin{array}{ll}
- & \text { Additive-free } \\
- & 0.01 \% \text { G-block } \\
\square- & 0.05 \% \text { G-block } \\
\square- & 0.1 \% \text { G-block } \\
\square & 0.1 \% \text { G-block (Na salt) }
\end{array}
$$

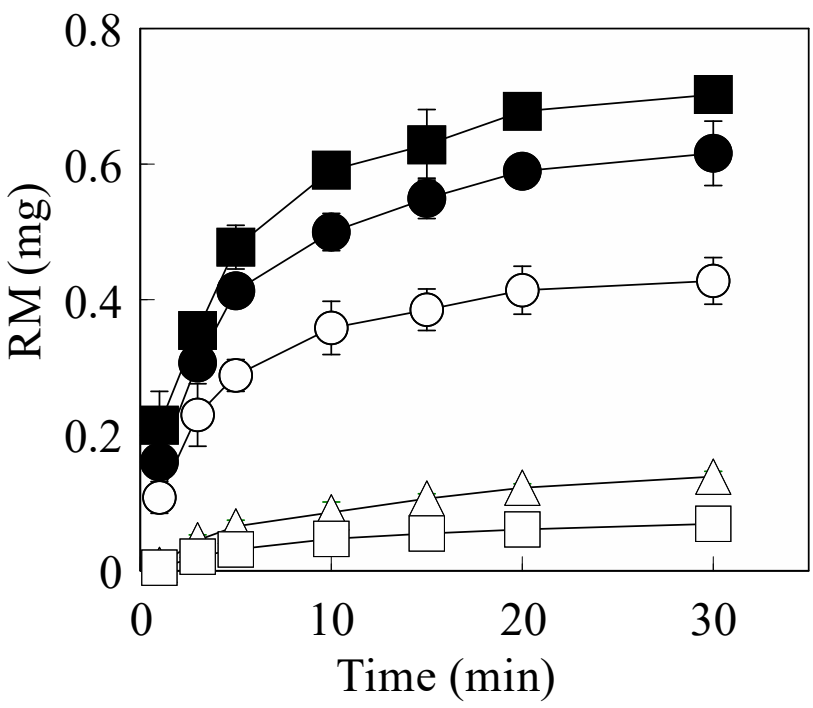

FIGURE 3: Drug release profiles of RM from FDs prepared with $2 \%$ Alg-C containing different concentrations and types of G-block. 
When G-block was added to the film base, the drug release rate from the FD decreased, as shown in Figure 2. The amount of RM that dissolved from the FD prepared with $4 \%$ Alg-B, containing $0.1 \%$ G-block, at $3 \mathrm{~min}$ was $0.13 \pm 0.04 \mathrm{mg}$. The drug dissolution rate was further reduced in the case of FD prepared with $4 \%$ Alg-B containing $0.2 \%$ G-block. However, the addition of $0.1 \%$ sodium salt of the G-block to the film base did not affect the drug dissolution rate. This phenomenon was observed in the FD prepared with $2 \%$ Alg-C. As shown in Figure 3, the drug dissolution rate decreased as the amount of additive incorporated into the FD increased. For example, the amount of RM that dissolved from the FD prepared with $2 \%$ Alg-C, containing $0.01 \%$ G-block, at 3 min was $0.23 \mathrm{mg}$. Furthermore, this amount decreased to $0.02 \mathrm{mg}$ for the FD prepared with 2\% Alg-C containing 0.1\% G-block.

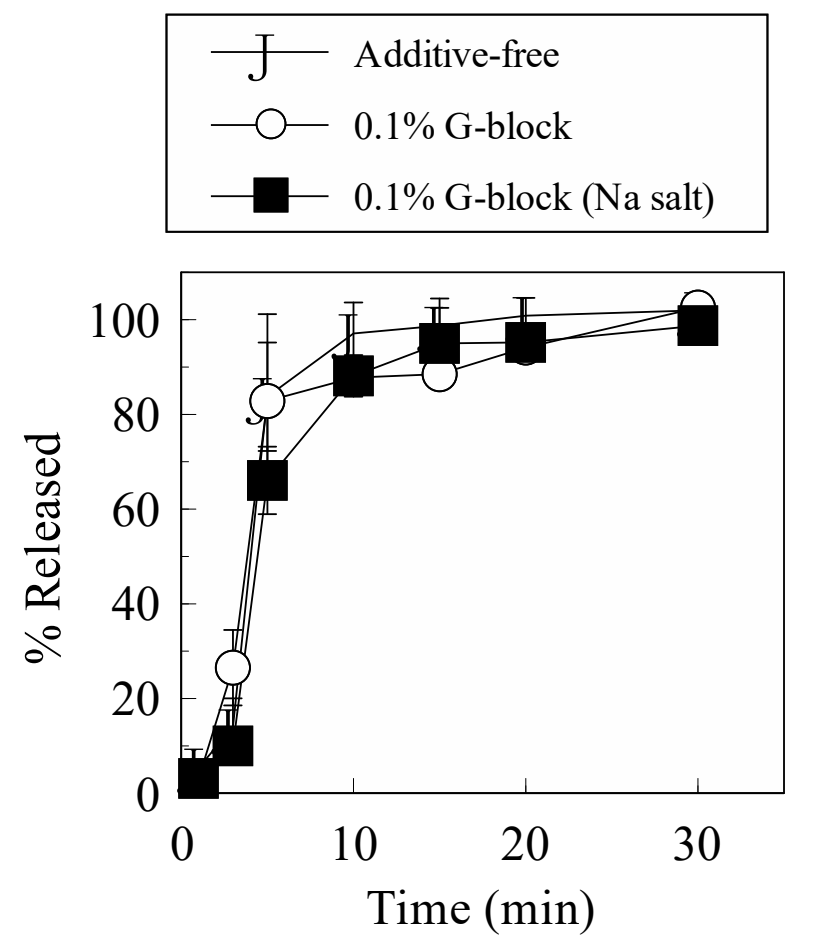

FIGURE 4: Dissolution profiles of Alg-Na from FDs prepared with 2\% Alg-C containing no additive or $0.1 \%$ additive.

The FD disintegrated in the drug dissolution test, resulting in the release of Alg-Na into the reaction solution. In this study, the amount of dissolved Alg-Na in the test solution was measured by a method that changed the uronic acid within the polysaccharide to a 
hydroxamic acid derivative. Figure 4 shows the disintegration profiles of the FD. For the FD prepared with $2 \%$ Alg-C, approximately $15 \%$ of the incorporated Alg-Na dissolved within 3 min, and the total amount of the film base dissolved into the test medium within $10 \mathrm{~min}$. A similar disintegration profile was observed for the FD prepared with 2\% Alg-C containing $0.1 \%$ G-block. These results show that the decrease in the drug dissolution rate after adding G-block is not caused by the suppression of the film matrix disintegration in an aqueous medium.

TABLE 1: Solubility of $R M$ in water containing $0.45 \%$ additive at $37^{\circ} \mathrm{C}$.

\begin{tabular}{|c|c|}
\hline Additive & Solubility $(\mu \mathrm{g} / \mathrm{mL})$ \\
\hline Additive-free & 170 \\
\hline Alg-A & 220 \\
\hline Alg-B & 4 \\
\hline G-block & 120 \\
\hline G-block (Na salt) & $<1$ \\
\hline Nicotinic acid & 250 \\
\hline Sodium nicotinate & $<1$ \\
\hline Aspartic acid & 150 \\
\hline Sodium aspartate & \\
\hline
\end{tabular}

Table 1 shows the water-solubility of $\mathrm{RM}$ at $37^{\circ} \mathrm{C}$. The solubility increased upon the addition of Alg-Na or the sodium salt of the G-block. In contrast, the addition of the G-block decreased the solubility of RM. This phenomenon was also observed when a weak acid, such as nicotinic acid and/or its sodium salt, was added to the solution. Figure 5 shows the drug release profiles of FDs prepared with Alg-Na containing a weak acid or its sodium salt. The 
amount of RM that dissolved from the FD prepared with $2 \%$ Alg-C, containing $0.1 \%$ nicotinic acid, at $5 \mathrm{~min}$ was $0.03 \mathrm{mg}$. In contrast, the amount of RM that dissolved from the film base containing $0.1 \%$ sodium nicotinate at $5 \mathrm{~min}$ was $0.48 \mathrm{mg}$. Based on these results, it can be assumed that the change in the RM dissolution rate from the FD is attributed to the interaction between the drug and components of the film base, such as Alg-Na or an additive.

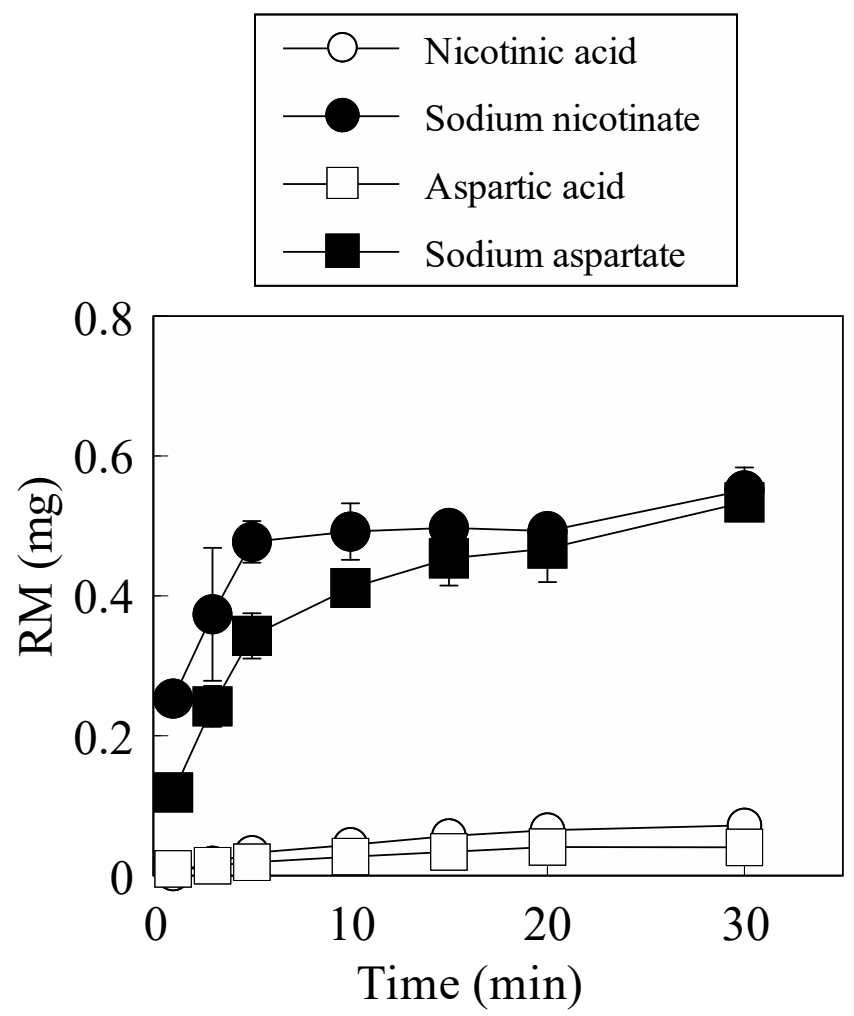

FIGURE 5: Drug release profiles of RM from FDs prepared with $2 \%$ Alg-C containing $0.1 \%$ additive.

\section{Conclusion}

Controlling the drug dissolution rate from FDs is an important factor in achieving efficient drug delivery to the disease site. In this study, we observed that the RM release rate from FDs prepared with Alg-Na can be controlled by modifying the film base with a G-block. RM has been recently approved as a therapeutic agent for oral mucositis or ulcers by local application. FDs containing RM prepared with Alg-Na may be an attractive dosage form for treating oral diseases. 


\section{Conflict of Interest}

The authors declare that there are no conflicts of interest regarding the publication of this manuscript.

\section{References}

[1] K. H. Min, S. F. Sasaki, Y. Kashiwabara, M. Umekawa, and K. Nisizawa, "Fine structure of SMG alginate fragment in the light of its degradation by alginate lyases of Pseudomonas sp,” Journal of Biochemistry, vol. 81, no. 3, pp. 555-562, 1977.

[2] F. Martínez-Gómez, A. Mansilla, B. Matsuhiro, M. C. Matulewicz, and M. A. Troncoso-Valenzuela, "Chiroptical characterization of homopolymeric block fractions in alginates," Carbohydrate Polymers, Vol. 146, pp. 90-101, 2016.

[3] Y. Murata, D. Jinno, D. Liu, T. Isobe, K. Kofuji, and S Kawashima, “The drug release profile from calcium-induced alginate gel beads coated with an alginate hydrolysate," Molecules, vol. 12, pp. 2559-2566, 2007.

[4] D. El. Khoury, H. D. Goff, and G. H. Anderson, "The role of alginates in regulation of food intake and glycemia: a gastroenterological perspective," Critical Reviews in Food Science and Nutrition, vol. 55, no. 10, pp. 1406-1424, 2015.

[5] S. Ścieszka, and E. Klewicka, "Algae in food: a general review," Critical Reviews in Food Science and Nutrition, vol. 59, no. 21, pp. 3538-3547, 2019.

[6] N. Uemura, I. Oda, Y. Saito, H. Ono, J. Fujisaki, N. Matsuhashi, K. Ohata, N. Yahagi, T. Yada, M. Satoh, H. Tajiri, M. Inomata, and S. Kitano, "Efficacy and safety of $0.6 \%$ sodium alginate solution in endoscopic submucosal dissection for esophageal and gastric neoplastic lesion: A randomized controlled study,” Digestive Endoscopy, vol. 31, no. 4, pp. 396-404, 2019.

[7] J. Wilkinson, .K Abd-Elaziz, I. den Daas, J. Wemer, M. van Haastert, V. Hodgkinson, M. 
Foster, and C. Coyle, "Two placebo-controlled crossover studies in healthy subjects to evaluate gastric acid neutralization by an alginate-antacid formulation (Gaviscon Double Action)," Drug Development and Industrial Pharmacy, vol. 45, no. 3, pp. 430-438, 2019.

[8] S. Sonmez, C. Coyle, D. Sifrim, and P. Woodland, "Duration of adhesion of swallowed alginates to distal oesophageal mucosa: implications for topical therapy of oesophageal diseases," Alimentary Pharmacology \& Therapeutics, vol. 52, no. 3, pp. 442-448, 2020.

[9] M. H. Jaafar, S. Z. Safi, M. P. Tan, S. Rampal, and S. Mahadeva, "Efficacy of rebamipide in organic and functional dyspepsia: a systematic review and meta-analysis," Digestive Diseases and Sciences, vol. 63, no. 5, pp. 1250-1260, 2018.

[10] H. Tanaka, T. Nakayama, M. Tsukamoto, A. Watanabe, T. Nakamura, N. Yokoi, C. Sotozono, and S. Kinoshita, "Rebamipide promotes lacrimal duct epithelial cell survival via protecting barrier function," Scientific Reports, vol. 10, no. 1, p. 1641, 2020.

[11] B. Chaitanya, K. M. Pai, P. H. Yathiraj, D. Fernandes, and Y. Chhaparwal, "Rebamipide gargle in preventive management of chemo-radiotherapy induced oral mucositis," Oral Oncology, vol. 72, pp. 179-182, 2017.

[12] S. Akagi, T. Fujiwara, M. Nishida, A. Okuda, Y. Nagao, T. Okuda, H. Tokuda, and K. Takayanagi, "The effectiveness of rebamipide mouthwash therapy for radiotherapy and chemoradiotherapy-induced oral mucositis in patients with head and neck cancer: a systematic review and meta-analysis," Journal of Pharmaceutical Health Care and Sciences, vol. 5, p. 16, 2019.

[13] A. O. Ferreira, M. A. F. Brandão, F. J. Raposo, H. C. Polonini, and N. R. B. Raposo, "Orodispersible films for compounding pharmacies," International Journal of Pharmaceutical Compounding, vol. 21, no. 6, pp. 454-461, 2017.

[14] R. G. Strickley, "Pediatric oral formulations: an updated review of commercially available pediatric oral formulations since 2007," Journal of Pharmaceutical Sciences, vol. 108, no. 4, pp. 1335-1365, 2019. 
[15] I. Takeuchi, C. Togo, and K. Makino, "Rebamipide-containing film using chitosan and HPMC for oral mucositis induced by cancer chemotherapy,” Anticancer Res, vol. 39, no. 12, pp. 6531-6536, 2019.

[16] Y. Murata, Y. Maejima, C. Maida, and K. Kofuji, "Disintegration properties and drug release profiles of chondroitin sulfate films," SCIREA Journal of Materials, vol.4, no. 2, pp. 32-40, 2019.

[17] Y. Murata, C. Maida, and K. Kofuji, "Drug release profiles and disintegration properties of pectin films," Materials vol. 12, no. 3, pp. 355-361, 2019.

[18] Y. Murata, C. Maida, and K. Kofuji, "Disintegration properties and drug release profiles of sodium alginate films containing rebamipide," Research \& Development in Material Science, vol. 15, no. 1, pp. 1635-1639, 2021.

[19] C. Gijare, and A. Deshpande, "Orodispersible films: a systematic patent review," Recent Patents on Drug Delivery and Formulation, vol. 12, no. 2, pp. 110-120, 2018.

[20] A. Haug, B. Larsen, and O. Smidsrod, "Uronic acid sequence in alginate from different sources," Carbohydrate Research, vol. 32, pp. 217-225, 1974.

[21] S. Sonawane, and P. Gide, "Optimization of forced degradation using experimental design and development of a stability-indicating liquid chromatographic assay method for rebamipide in bulk and tablet dosage form," Scientia Pharmaceutica, vol. 79, no. 1, pp. 85-96, 2011. 